\title{
Are the IKKs and IKK-related kinases TBK1 and IKK- $\varepsilon$ similarly activated?
}

\author{
Tieu-Lan Chau ${ }^{1 *}$, Romain Gioia ${ }^{1 *}$, Jean-Stéphane Gatot ${ }^{1}$, Félicia Patrascu ${ }^{1}$, \\ Isabelle Carpentier ${ }^{2}$, Jean-Paul Chapelle ${ }^{1}$, Luke O'Neill $^{3}$, Rudi Beyaert ${ }^{2}$, \\ Jacques Piette ${ }^{1}$ and Alain Chariot $^{1}$
}

\author{
${ }^{1}$ Interdisciplinary Cluster for Applied Genoproteomics, Medical Chemistry, and Virology/lmmunology units, \\ University of Liège, Sart-Tilman, 4000 Liège, Belgium \\ ${ }^{2}$ Unit of Molecular Signal Transduction in Inflammation, Department for Molecular Biomedical Research, VIB, Ghent, Belgium \\ ${ }^{3}$ School of Biochemistry and Immunology, Trinity College, Dublin, Ireland
}

\begin{abstract}
The IKB kinases (IKKs) IKK- $\alpha$ and IKK- $\beta$, and the IKK-related kinases TBK1 and IKK- $\varepsilon$, have essential roles in innate immunity through signal-induced activation of NF- $k$ B, IRF3 and IRF7, respectively. Although the signaling events within these pathways have been extensively studied, the mechanisms of IKK and IKK-related complex assembly and activation remain poorly defined. Recent data provide insight into the requirement for scaffold proteins in complex assembly; NF-кB essential modulator coordinates some IKK complexes, whereas TANK, NF-кB-activating kinase-associated protein 1 (NAP1) or similar to NAP1 TBK1 adaptor (SINTBAD) assemble TBK1 and IKK- $\varepsilon$ complexes. The different scaffold proteins undergo similar post-translational modifications, including phosphorylation and non-degradative polyubiquitylation. Moreover, increasing evidence indicates that distinct scaffold proteins assemble IKK, and potentially TBK1 and IKK- $\varepsilon$ subcomplexes, in a stimulus-specific manner, which might be a mechanism to achieve specificity.
\end{abstract}

the expression of a variety of cell surface markers and their responses to pathogen molecules. pDCs are defined as a subset of cells, the appearance under the microscope of which is similar to that of plasmablasts. These cells are the main producers of type I IFNs in response to viral infections. CpG DNAs: CpG DNAs are DNA oligodeoxynucleotide sequences that include a cytosine-guanosine sequence and some flanking nucleotides. The CpG DNAs induce innate immunity through binding to the TLR9 receptor.

Cytosolic NF-KB and IRF activating pathways: these pathways include the RIG-I family (comprising MDA5 and RIG-I) and are triggered following infection with RNA viruses and also the DAl-dependent pathway, which is activated when this cytosolic receptor senses DNA from viruses or damaged cells.

E3 ligase: E3 ligases are defined as enzymes that facilitate the transfer of the ubiquitin from the ubiquitin-conjugating enzyme (E2) to the $\varepsilon$-amino group of a Iysine residue in a target protein.

Innate immunity: innate immunity is defined as the initial, rapidly induced immune response of most multicellular organisms. This immunity relies on receptors required for pathogen recognition and the recruitment and activation of phagocytic cells.

Interferons (IFNs): IFNs are defined as cytokines that block viral replication and infection of surrounding cells.

IFN-regulatory factors (IRFs): IRFs are a family of nine proteins (IRF1 to IRF9) that share a well-conserved DNA-binding domain of $\sim 120$ amino acids at their $\mathrm{N}$-terminus. This domain is required to bind to the consensus DNA sequence that is known as the ISRE. IRFs are involved in the development and function of

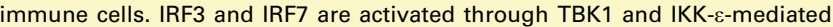
phosphorylation on their C-terminal domain.

IFN-stimulated response element (ISRE): ISRE is a DNA motif that is bound by IRFs. The consensus sequence is GAAANNGAAAG/CT/C, where $\mathrm{N}$ denotes any nucleotide.

Lipopolysaccharide (LPS): LPS is a component of the outer membrane of Gram-negative bacteria and triggers NF- $\mathrm{KB}$ and IRF activation through binding to the TLR4 receptor.

NF-KB essential modulator (NEMO): NEMO is required to assemble IKK- $\alpha$ and IKK- $\beta$ into an I $\mathrm{K} \beta \alpha$-phosphorylating complex following stimulation by various stimuli, including proinflammatory cytokines and molecular components of pathogens.

Nuclear factor (NF)-кB: NF- $\mathrm{kB}$ is a structurally and evolutionarily conserved family of transcription factors initially identified as proteins harboring a DNAbinding activity for the enhancer of the immunoglobulin $\kappa$ light-chain in activated $B$ cells. These proteins are crucial for the production of proinflammatory cytokines, growth factors and enzymes required for the initiation and resolution of the immune response. These proteins include RelA (also known as $\mathrm{p} 65$ ), RelB, c-Rel and also $\mathrm{p} 50$ and $\mathrm{p} 52$ (which are generated from processed precursors - namely, p105 and p100, respectively).

Pathogen-associated molecular patterns: a molecular pattern which is found in microorganisms but not in host cells.

Scaffold protein: a scaffold protein is referred to as a molecule that functions as a platform in order to promote the recruitment and assembly of complexes. These proteins do not harbor any enzymatic activity but are nevertheless essential for the activation of the enzymes to which they bind.

Toll-IL-1 receptor (TIR) domain: the TIR domain is defined as an amino acid sequence of the cytoplasmic region that is highly conserved among members of the TLR and IL-1 receptor superfamily.

Type I IFNs: a family of proteins that includes IFN- $\alpha$ and IFN- $\beta$.

Ubiquitylation: the post-translational modification of proteins by the attachment of one or more $7 \mathrm{kDa}$ ubiquitin molecules to lysine residues of the substrates. This modification triggers protein degradation (K48-linked polyubiquitylation) or is required for cell signaling (K63-linked polyubiquitylation). 


\section{The NF-кB- and IRF-activating signaling pathways in innate immunity}

The innate immune system (see Glossary) senses a variety of pathogen-associated molecular patterns (PAMPs), such as bacterial lipopeptides, viral and/or bacterial nucleic acids, by means of specific receptors. As a consequence, genes encoding the type I interferons (IFNs) IFN- $\alpha$ and $-\beta$, proinflammatory cytokines [e.g. tumor necrosis factor- $\alpha$ (TNF- $\alpha)$ and interleukin-1 $\beta$ (IL-1 $\beta$ )] and chemokines [e.g. IL-8, monocyte chemoattractant protein-1 (MCP-1)] are induced [1,2]. Signaling pathways triggered by these viral or bacterial products occur through the Toll-like receptor (TLR) [3-5] or the cytosolic receptor pathway termed the retinoid-acid-inducible gene I (RIG-I)-dependent pathway [1,6]. Both pathways rely on the coordinated activation of transcription factors such as nuclear factor-кB (NF-кB) and IFN regulatory factors (IRFs) $[7,8]$.

Both the NF- $\mathrm{KB}$ and IRF families of transcription factors are simultaneously activated in response to viral or bacterial infection but the target genes that are ultimately induced through these signaling pathways are distinct. Indeed, whereas the induction of proinflammatory cytokines requires $\mathrm{NF}-\kappa \mathrm{B}$, type I $I F N$ gene induction mainly relies on IRF activation [2,7,8].

$\mathrm{NF}-\kappa \mathrm{B}$ activation following viral or bacterial infections involves the signal-induced phosphorylation and subsequent degradative polyubiquitylation of the inhibitory $\kappa \mathrm{B} \alpha$ protein $\left(\mathrm{I}_{\kappa} \mathrm{B} \alpha\right)$ through the canonical IкB kinase (IKK)-dependent pathway $[9,10]$. This IKK complex contains the catalytic subunits IKK- $\alpha$ and IKK- $\beta$ and the scaffold protein NF- $\mathrm{KB}$ essential modulator (NEMO; also called IKK- $\gamma$ ) [11-13]. Optimal NF-кB activity also requires signal-induced phosphorylations of the NF-кB protein $\mathrm{p} 65$ [14]. In contrast to NF- $\mathrm{KB}$ activation, which relies on the degradation of a cytoplasmic inhibitory protein and subsequent release of 'free' or 'activated' NF$\kappa \mathrm{B}$ proteins, IRF3 and IRF7 activation in the cytoplasm occurs directly through their C-terminal phosphorylation by two kinases, namely TNF-receptor-associated factor (TRAF) family member-associated NF- $\mathrm{B}$ activator (TANK)-binding kinase [TBK1; also referred to as NFкB-activating kinase (NAK) [15] or TNF-receptor-associated factor 2 (TRAF2)-interacting kinase (T2K) [16]] and IKK- $\varepsilon$ (also referred to as IKK-i [17]) $[18,19]$. These modifications promote IRF3 and IRF7 homodimerization and their subsequent nuclear import (Figure 1).

Importantly, many, if not all, cytoplasmic kinases require assembly by scaffold proteins to target and phosphorylate their substrates efficiently. Whereas NEMO assembles some but not all IKK complexes [12,13], recent reports provide strong experimental evidence for a role of TANK [also called TRAF-interacting protein (I-TRAF)], NAK-associated protein (NAP1) and similar to NAP1 TBK1 adaptor (SINTBAD) in the assembly of TBK1 and IKK- $\varepsilon$ kinase complexes that phosphorylate IRF3 and IRF7 and promote type I IFN gene induction [20-24].

Here, we describe the TBK1- and IKK- $\varepsilon$-dependent signaling pathways and address the biological roles of their scaffold proteins TANK, NAP1 and SINTBAD in the signaling pathways required for establishment of innate immunity. We compare and contrast their modes of action and discuss the unresolved issues in this field of research.

\section{Which scaffold proteins assemble IKK- $\alpha$ and IKK- $\beta$ ?}

The NF-кB-activating signaling pathways that are triggered following stimulation by TLR ligands ultimately converge upon the classical IKK complex that includes IKK- $\alpha$ and IKK- $\beta$ as catalytic subunits and the protein NEMO [10]. This latter IKK subunit is viewed as a unique and essential scaffold protein required for the assembly of this kinase complex in these pathways $[12,13,25]$. Interestingly, other stimuli, such as lymphotoxin- $\beta$, B cell-activating factor belonging to the TNF family (BAFF) and CD40L, trigger the alternative pathway through an IKK- $\alpha$-dependent, but IKK- $\beta$ - and NEMO-independent, pathway [26-28]. This signaling cascade relies on NFкB-inducing kinase (NIK), the activation of which triggers phosphorylation of the inhibitory protein p100 through an IKK- $\alpha$-dependent pathway [29-31]. This signaling pathway presumably relies on a yet to be identified scaffold protein that assembles a functional IKK- $\alpha$ homodimer. Thus, specificity within the NF-кB-activating pathways can be achieved through the use of signal-specific upstream kinases - that is, transforming growth factor- $\beta$-activating kinase (TAK1) or NIK for the classical or the alternative pathways, respectively - which ultimately target different IKK subcomplexes that have been assembled by distinct scaffold proteins. Whether this paradigm applies only to the NF-кB-activating pathways remains unclear but recent data indicate that this is not the case [21].

\section{What are the TBK1- and IKK- $\varepsilon$-dependent signaling pathways?}

Whereas the NF-кB-activating and NEMO-dependent pathways were quickly revealed based on the characterization of NEMO-deficient mice or cells [12,25], the signaling pathways that specifically rely on TBK1 and IKK- $\varepsilon$ remained elusive for many years. TBK1 and IKK- $\varepsilon$ were initially described as NF-кB-activating kinases, based on the fact that mice deficient for $t b k 1 / t 2 k$ died from massive liver apoptosis in utero [16], similarly to the mice deficient for $p 65, I K K-\beta$ or NEMO [25,32-35], and on the ability of TBK1 to target IKK- $\beta$ in vitro [15]. However, subsequent studies disclosed a role for TBK1 as an IKK-activating kinase [36,37]. Indeed, the phenotypical characterization of $t b k 1$-deficient cells undoubtedly highlighted a crucial role for TBK1 in $I F N$ gene induction $[36,37]$ through a signal-induced phosphorylation of IRF3 and IRF7 by this IKK-related kinase $[18,19]$. These results defined both TBK1 and IKK- $\varepsilon$ as IRF3 and IRF7 kinases that sense viral and bacterial infections through multiple signaling pathways.

Several stimuli that direct TBK1- and IKK- $\varepsilon$-dependent IRF3 and IRF7 phosphorylations have been identified. These include lipopolysaccharide (LPS), a component of the outer membrane of Gram-negative bacteria, and double-stranded (ds) RNA, a product of many replicating viruses, which trigger the TLR4- or the TLR3-dependent signaling cascade, respectively [3] (Figure 1). Both pathways requires Toll-IL-1 receptor (TIR) domain-containing adaptor inducing IFN- $\beta$ (TRIF) [also called TIR-containing 


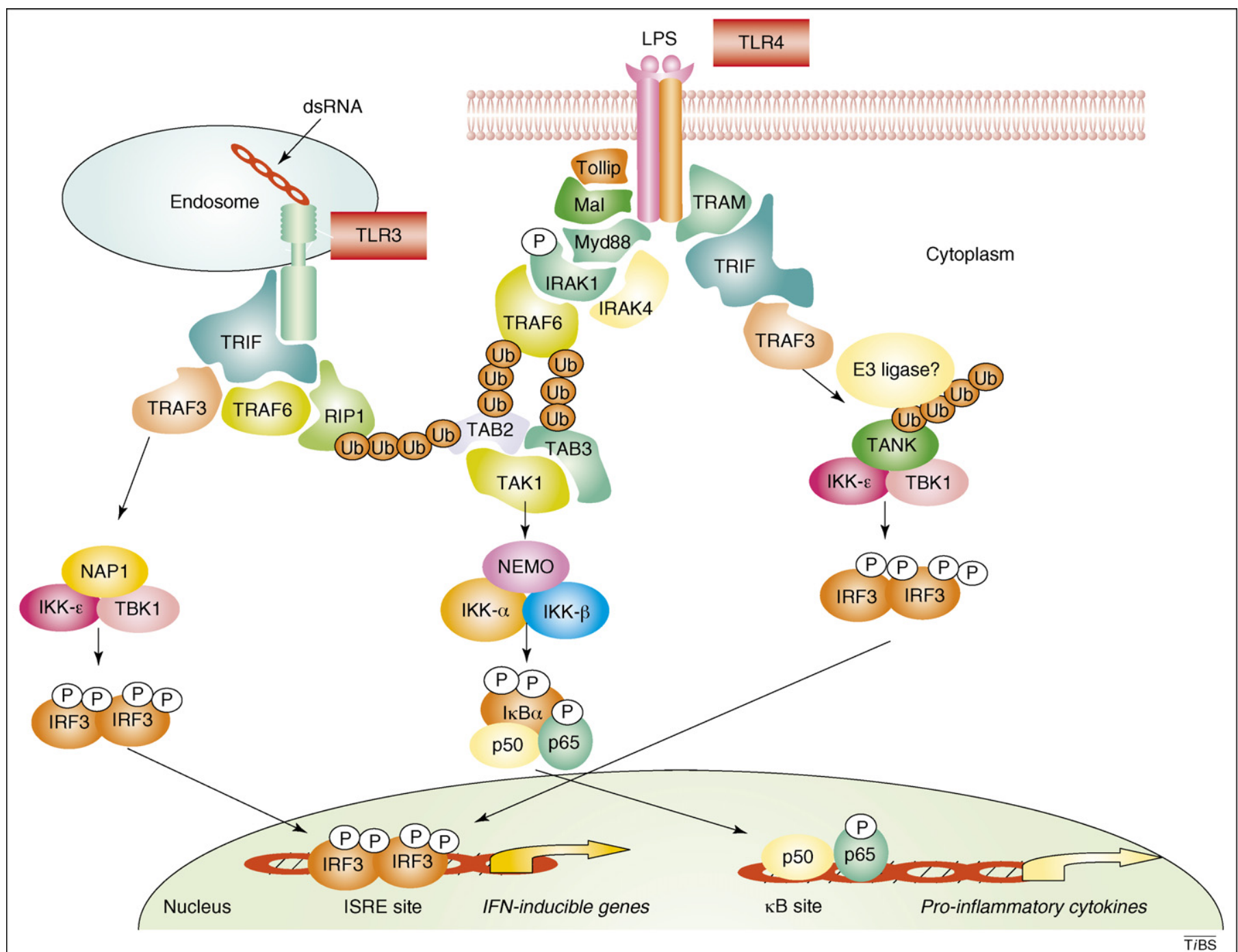

Figure 1. TLR3 and TLR4-dependent signaling pathways. TLR3-mediated pathways are triggered following dsRNA binding. TLR3 (green) is localized to the cell surface in fibroblasts and to endosomes in conventional dendritic cells. The TIR domain-containing TRIF adaptor (turquoise) binds to the TLR3 TIR domain; this binding is required for subsequent IRF3 (orange) and NF-KB activation. The IRF3-activating pathway also involves TRAF3 (light orange); this connects TRIF to the TBK1-IKK- $\varepsilon$ (TBK1: pink; IKK- $\varepsilon$ : red) heterodimer kinase complex, which is assembled by the scaffold protein NAP1 (gold). IRF3 is phosphorylated (P) within its C-terminal domain by TBK1-IKK- $\varepsilon$ and forms a homodimer which translocates to the nucleus, binds to ISREs and induces the expression of IFN-dependent genes. The TLR3-dependent NF-KB-activating pathway relies on RIP1 (green) and also on the E3 ubiquitin ligase TRAF6 (light green), which exhibits a cell type-specific essential role in this signaling cascade. RIP1 and TRAF6 are recruited to TRIF, and the signal-induced polyubiquitylated RIP1 is subsequently recruited to the TAB2-TAK1 (TAB2: light blue; TAK1: light green) signaling complex, a crucial step for IKK activation (IKK- $\alpha$ : orange; IKK- $\beta$ : blue). The IKK complex is assembled by NEMO (pink) and targets IKB $\alpha$ (orange) for phosphorylation and subsequent degradative polyubiquitylation. The $p 50-p 65$ heterodimer ( $p 50$ : yellow; $p 65$ : green) is then released from $1 \kappa B \alpha$, moves into the nucleus and induces the expression of various proinflammatory genes through binding to $\mathrm{KB}$ sites. Of note, optimal NF- $\mathrm{KB}$ activation also requires signal-induced $\mathrm{p} 65$ phosphorylation. LPS binding to the TLR4 receptor also triggers NF-KB and IRF activation through distinct adaptor proteins. NF- $\mathrm{KB}$ activation relies on the TIR domain-containing adaptors Mal (green) and Myd88 (light blue), whereas LPS-mediated IRF3 activation relies on TRAM (light blue) and TRIF. IRF3 activation also requires TRAF3, which binds to TANK (green), the scaffold

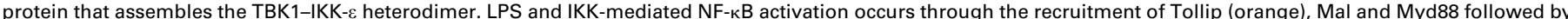
the binding of the kinase IRAK1 (light blue) to TLR4. IRAK1 is subsequently phosphorylated by IRAK4 (yellow). This event triggers the recruitment of TRAF6 to the receptor complex and subsequent signal-induced association of these proteins with the TAB2-TAB3-TAK1 complex, a step required for IKK activation.

adapter molecule-1 (TICAM-1)] [38,39], one of the five TIRdomain-containing adaptors identified so far [40]. TRAF3, which connects TRIF to the TBK1 and IKK- $\varepsilon$ kinase complexes for subsequent IRF3 phosphorylation, lies downstream of TRIF [41-44]. The TLR7, TLR8 and TLR9 receptors, which, in contrast to TLR4, are not localized on the cell surface but rather in endosomal compartments, sense viral nucleic acids in plasmacytoid dendritic cells and also elicit IFN gene induction through IRF7 phosphorylation [45]. However, TBK1 and IKK- $\varepsilon$ do not seem to be required for these pathways [46]. Instead, IRF7 phosphorylation is IKK- $\alpha$ dependent $[45,47]$.
IRF3 and IRF7 are not exclusively phosphorylated through the TLR-dependent pathways. Indeed, establishment of the innate immune response to viral pathogens also relies on the so-called cytosolic RIG-I and melanoma differentiation-associated gene 5 (Mda5)-dependent pathway both in fibroblasts and in conventional dendritic cells [6]. Both proteins harbor a $\mathrm{DEx}(\mathrm{D} / \mathrm{H})$ box RNA helicase domain that is required to sense dsRNA synthesized from replicating virus entering the cytoplasm (e.g. the Sendai virus, the Newcastle disease virus or the vesicular stomatitis virus [6]) (Figure 2). Because they also harbor two caspase-recruitment domain (CARD)-like domains, they 


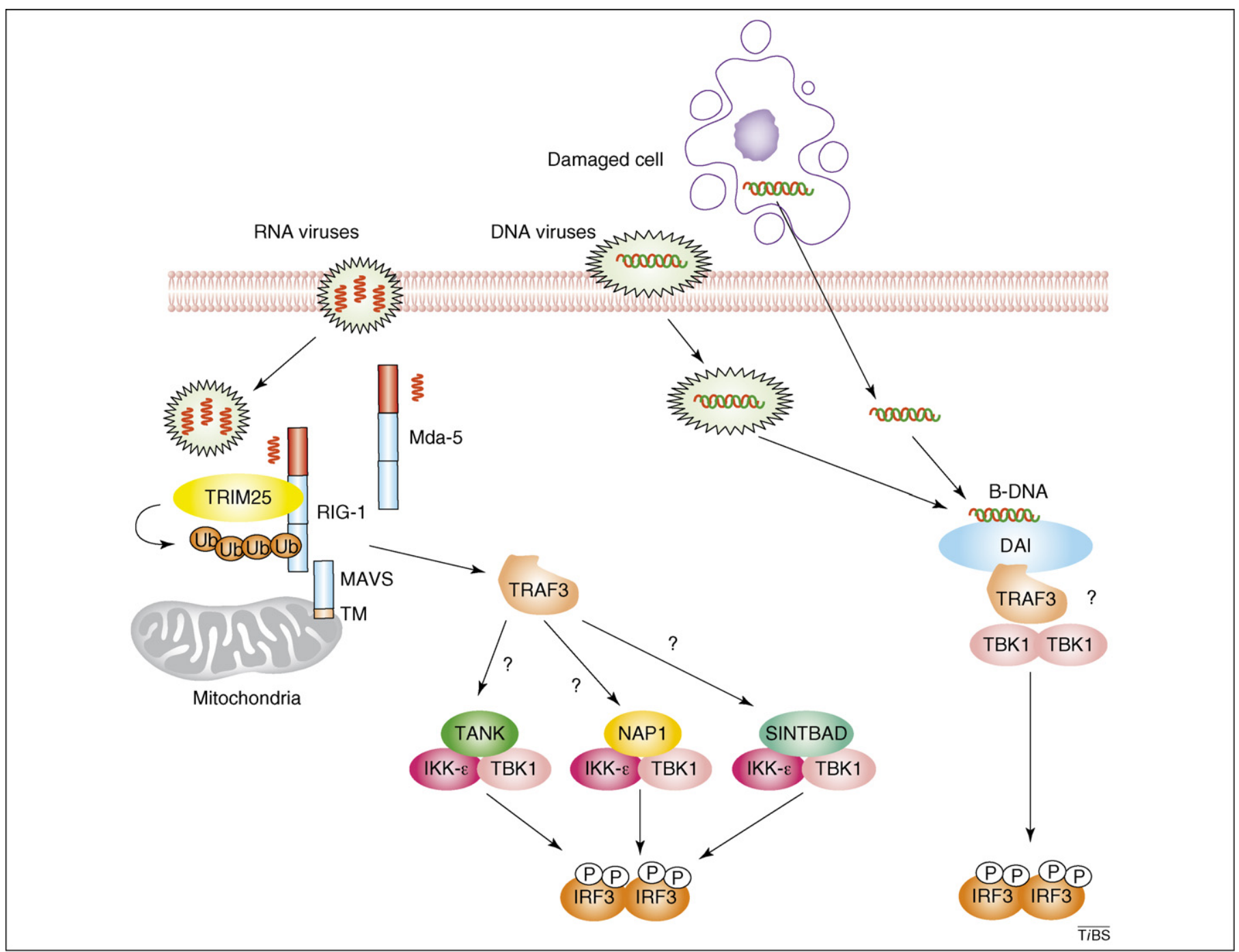

Figure 2. The cytosolic, TBK1- and IKK- - -dependent signaling pathways. IRF3 activation is triggered when cytosolic receptors sense intracellular nucleic acids (RNA from viruses or DNA from viruses or damaged cells). RNA from viruses triggers the activation of the cytosolic receptors RIG-I and MDA-5 through binding to their DExD/H box RNA helicase (red boxes). The CARD domains of these receptors (light blue boxes) subsequently undergo K63-linked polyubiquitylation by the E3 ubiquitin ligase TRIM25 (yellow), which might facilitate the interaction of RIG-I with the mitochondrial adaptor MAVS. Importantly, MAVS harbors a mitochondrial transmembrane domain (TM)

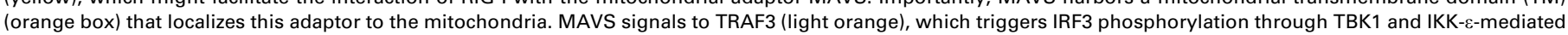
activations. Of note, MAVS can also signal to TRAF6 for the IKK- $\alpha-I K K-\beta$-complex-mediated NF-kB activation but this pathway is not represented. TANK (green), NAP1 (gold)

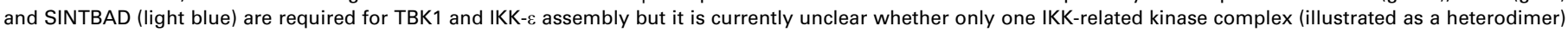
assembled by multiple scaffold proteins is involved or whether distinct subcomplexes assembled by only one scaffold protein (i.e. TANK, NAP1 or SINTBAB) are simultaneously activated through this cytosolic pathway, as illustrated here. These adaptors might also be targeted for K63-linked polyubiquitylation but this hypothesis

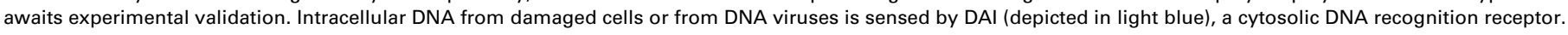
Activated DAI binds to TBK1 (represented as a homodimer) and triggers IRF3 phosphorylation. It is currently unknown whether TRAF3 is part of this signaling complex. The adaptors for TBK1 homodimers remain to be elucidated.

can transmit the signal through direct binding to mitochondrial antiviral signaling (MAVS) [48] [also called IFN$\beta$ promoter stimulator 1 (IPS-1), virus-induced signaling adaptor (VISA) and Cardif [49-51]), a CARD domain-containing mitochondrial adaptor. This protein subsequently triggers NF-кB activation through TRAF6 and the IKK- $\alpha-$ $\beta$ complex [50] or IRF3 and IRF7 activation through TRAF3 and the TBK1 and IKK- $\varepsilon$ kinase complexes [48]. Intracellular dsDNA from microbes or damaged cells also modulates the innate immune response by triggering TBK1 and IKK- $\varepsilon$ activation [52] but the cytosolic receptor required in this pathway is not RIG-I. Indeed, the intracellular DNA sensor is DNA-dependent activator of IFNregulatory factors (DAI), which binds to, and is activated by, DNA from various sources. DAI subsequently recruits
TBK1, which triggers IRF3 phosphorylation and type I IFN gene expression [53].

\section{Which scaffold proteins assemble the IKK-related kinases TBK1 and IKK- $\varepsilon$ ?}

Similar to the IKKs, TBK1 and IKK- $\varepsilon$ require assembly by a scaffold protein to target their substrates efficiently. These scaffold proteins must constitutively interact with the catalytic subunits of the complex, and three candidates, TANK, NAP1 and SINTBAD, seem to fulfill this function.

\section{TANK}

TANK was originally identified as a TRAFinteracting protein that synergizes with TRAF2 to induce 
NF-кB-dependent reporter gene expression [54-56]. Later, its role in the $\mathrm{NF}-\mathrm{\kappa B}$-activating pathways was supported by its binding to NEMO [57]. However, the role for TANK in the IKK-dependent NF- $\mathrm{kB}$ signaling cascades remains controversial because TANK-depleted, knockdown cells do not show any defect in TNF- $\alpha$-or LPSmediated IKK activation [21,58]. Nevertheless, these observations do not rule out the possibility that TANK might connect upstream kinases such as TBK1 and IKK- $\varepsilon$, thereby promoting IKK-independent phosphorylation of the NF-кB proteins p65 [59,60], c-Rel [61] or p52 [62] in response to as yet poorly characterized signals. TANK constitutively binds to IKK- $\varepsilon$ [63] and TBK1 [64] through its $\mathrm{N}$-terminal domain; this interaction is probably essential for the positive regulation of signal transduction by TANK because recent studies demonstrated that TANK is involved in some TLR-dependent IRF-activating pathways by promoting TBK1 and IKK- - -mediated phosphorylation of IRF3 and IRF7 [20,21].

\section{NAP1}

NAP1, a candidate scaffold protein that was initially identified as a TBK1-interacting protein, shares several structural features with TANK [65]. Early studies indicated that NAP1 assembles TBK1 complexes for subsequent p65 phosphorylation and therefore has a role in the NF-кB-activating pathways [65]. Enhanced sensitivity to apoptosis of the TNF- $\alpha$-stimulated, NAP1-depleted knockdown cells, which is typical for cells having defects in the prosurvival NF- $\mathrm{kB}$ signaling cascade, further supported this hypothesis [65]. Similarly to TANK, NAP1 constitutively binds to TBK1 and is also required for IRF3 phosphorylation through both the TLR3- and the RIG-I-dependent pathways [22,23].

\section{SINTBAD}

SINTBAD is the most recently identified scaffold protein that constitutively binds to TBK1 and IKK- $\varepsilon$ [24]. Interestingly, this protein shares a conserved TBK1- and IKK- $\varepsilon$ binding domain (TBD) with TANK and NAP1, and this region is predicted to form an $\alpha$-helix with the conserved residues clustering on one side of it [24]. Owing to the structural similarities between TANK, NAP1 and SINT$\mathrm{BAD}$, it is expected that they perform similar functions in TBK1 and IKK- $\varepsilon$ activation.

\section{TANK-, NAP1- and SINTBAD-dependent signaling pathways}

In addition to TANK and NAP1 possibly regulating NF- $\mathrm{kB}$ activation through p65-mediated phosphorylation, both scaffold proteins are also required for IRF3 and IRF7 phosphorylation because they constitutively bind to TBK1 and IKK- $\varepsilon$. Only recently have studies started to explore TBK1- and IKK $\varepsilon$-dependent pathways in which TANK, NAP1 and SINTBAD are specifically required. Interestingly, some specificity at the level of TBK1 and IKK- $\varepsilon$ assembly might occur because NAP1 is essential for the TLR-mediated IRF3 activation pathway [22], whereas TANK seems to have a similar role for IRF3 phosphorylation through the TLR4 pathway in LPS-responsive cells such as macrophages [21] (Figure 1). In agreement with this hypothesis, the early phase of dsRNA-mediated IRF3 phosphorylation through the TLR3 receptor does not require TANK [21]. Thus, these results indicate that TANK functions as a scaffold protein that assembles some, but not all, IRF3- and IRF7-phosphorylating TBK1 and IKK- $\varepsilon$ complexes. In other words, this observation opens the possibility that distinct scaffold proteins might be required for TBK1 and IKK- $\varepsilon$ assembly in a pathwayspecific manner. The dispensable role of TBK1 and IKK$\varepsilon$ in the TLR7-, TLR8- and TLR9-dependent IRF7-activating pathways makes an involvement of TANK and NAP1 in these cascades unlikely. Of note, however, IRF7 was also identified as a TANK-interacting protein through yeast two-hybrid analyses, and IRF7 can be phosphorylated by TANK-containing immune complexes in macrophages treated with unmethylated CpG DNA motifs, which are TLR9 ligands [21]. These data indicate that TANK might also have a role in this IRF7-dependent signaling cascade by connecting a yet to be identified kinase to this IRF member. Alternatively, TANK might connect TBK1 and/or IKK- $\varepsilon$ to IRF7 in a second wave of activation through the TLR4, a hypothesis supported by the LPS-inducible expression of TANK, IKK- $\varepsilon$ and IRF7 $[17,66]$.

The signaling pathways that involve TANK, NAP1 and SINTBAD do not exclusively include the TLR-dependent signaling pathways. Indeed, the cytosolic RIG-Idependent pathway, which triggers TBK1 and IKK- $\varepsilon$ activations, also requires these scaffold proteins (Figure 2). TANK is known to be one candidate because TANK-depleted knockdown cells infected with Sendai virus exhibit defective IFN- $\beta$ production [20]. Such a model is further supported by the virus-inducible TANK-MAVS association in fibroblasts [20]. Moreover, NAP1 and SINTBAD depletion through RNA interference also causes defects in Sendai virus-induced activation of an IFN-stimulated responsive elements (ISRE)containing reporter construct [24]. Thus, these results indicate that TANK, NAP1 and SINTBAB are all required for type I IFN induction through the cytosolic receptor and IRF3-activating pathways.

\section{Crosstalk between the NF-кB and IRF pathways}

Crosstalk is a common feature in signal transduction and also seems to be relevant for the NF-кB- and IRF-activating pathways (Figure 3 ). This hypothesis is supported by the physical association between TANK and NEMO $[57,67]$. The consequence of this crosstalk remains unclear but might underlie the molecular basis for the generation of specific IRF3-p65 complexes which are required for the proper expression of a subset of genes in LPS-stimulated cells [68-70]. Additional evidence for crosstalk between the two signaling pathways is provided by IKK- $\varepsilon$-mediated phosphorylation of p65 in TNF- $\alpha$-stimulated cells [71]. However, it still remains unclear whether the transcriptional complexes involving both the IRF and NF-кB proteins require a physical association between the IKK and IKK-related scaffold proteins (e.g. between NEMO and TANK) or whether such heterodimers are generated following simultaneous, but independent, activation of the pathways through the TLR- or RIG-I-dependent pathways. Of note, some signaling molecules are involved in 


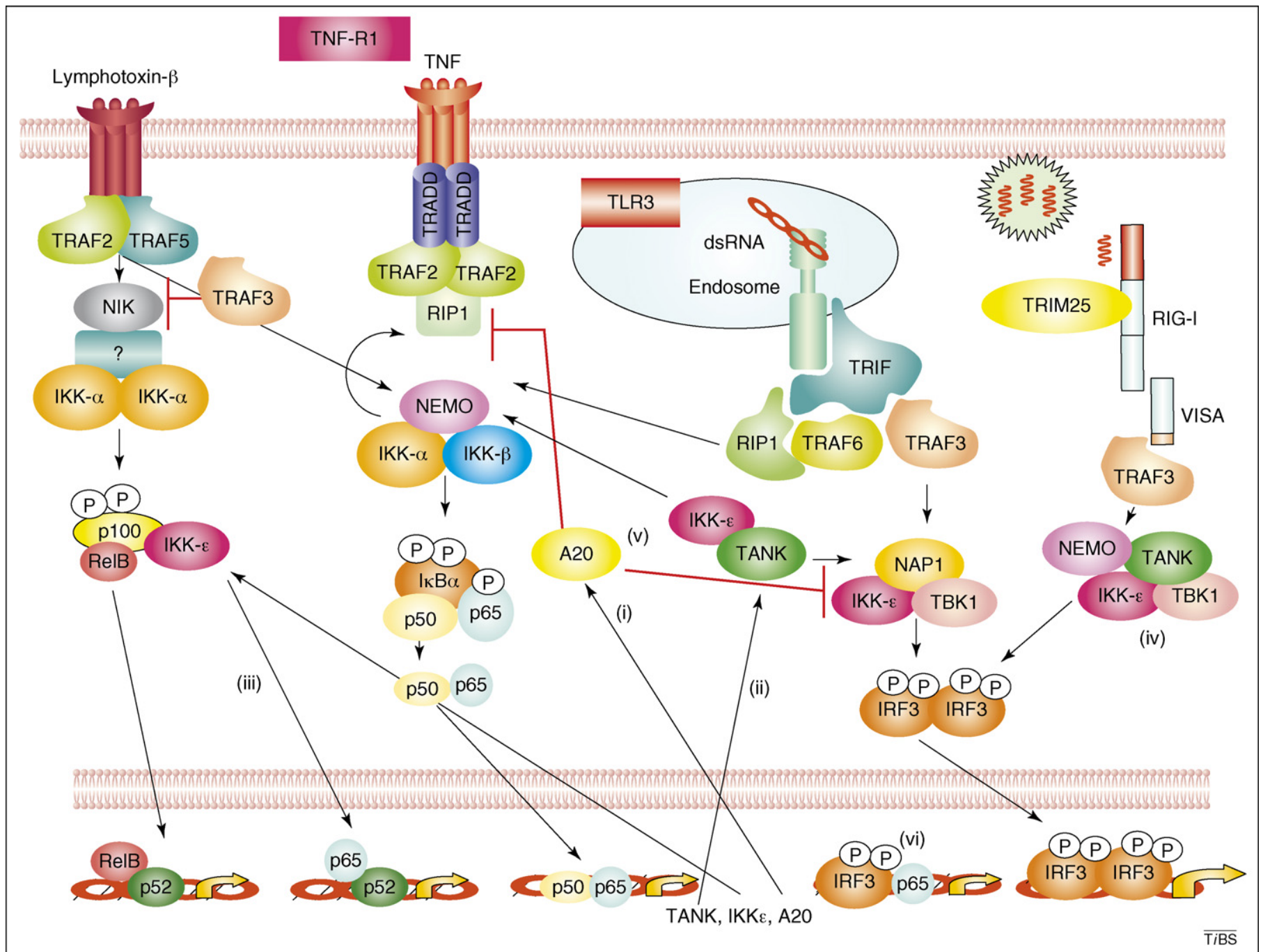

Figure 3. Crosstalk between the NF-кB and IRF pathways. Both the NF-kB- and IRF-dependent pathways share common signaling molecules which have crucial roles in the crosstalk between these cascades. The first crosstalk involves the NF-kB-dependent transcriptional induction of several proteins (TANK, IKK- $\varepsilon$ and A20), which ultimately interfere with the p65- or the IRF3-activating cascades (i). TNF- $\alpha$ triggers NF-kB activation through TRADD (blue) and the classical, NEMO-dependent pathway, which

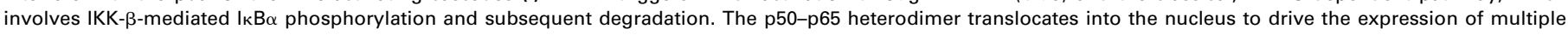
genes, including TANK (green), IKK- $\varepsilon$ (pink) and A20 (yellow). A20 subsequently turns off NF-KB activation (red lines) by targeting RIP1 (light green) for a K48-linked degradative polyubiquitylation step (i). A20 also negatively regulates the TLR3-mediated IRF3 activation cascade through direct binding to the TBK1 signaling complex. TANK is also transcriptionally induced and participates in later phases of IRF3 activation following infection by RNA viruses through the TLR3-dependent pathway (ii). A second crosstalk is established between IKK- $\varepsilon$ and p100 (yellow), an inhibitory molecule whose processing into p52 (light red) is triggered following stimulation with

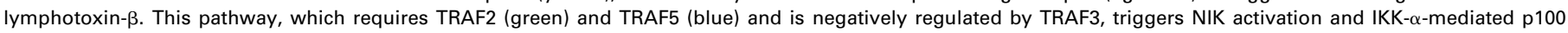
phosphorylation. This IKK complex is assembled by a yet to be identified scaffold protein ('?'). p100 is subsequently processed into p52 and moves into the nucleus as a heterodimer with the NF-KB protein RelB (red). IKK- $\varepsilon$ expression is also induced in TNF- $\alpha$-stimulated cells, and this kinase associates with p100 and p52 to enhance the transactivation potential of specific p52-p65 heterodimers (iii). A third crosstalk occurs through direct interactions between scaffold proteins. For example, NEMO can be part of an IRF3-phosphorylating, TANK-containing complex following viral infection and subsequent activation of the RIG-I-dependent pathway (iv). TANK also connects IKK- $\varepsilon$ and TBK1 (not illustrated) to the IKK complex through binding to NEMO for subsequent p65 phosphorylation (v). Finally, a fourth crosstalk occurs in the nucleus, where the NF-кB and IRF3 proteins can be part of common transcriptional complexes. Indeed, some IRF3-p65 complexes (vi) are specifically generated following signaling through the TLR4- (not represented for clarity purposes) but not the TLR3-dependent pathway.

both pathways but differentially regulate them. For example, the TNF- $\alpha$-inducible A20 protein negatively regulates both NF-кB and IRF3-activating pathways by promoting the K48-linked, degradative polyubiquitylation of the receptor-interacting protein (RIP1) in TNF- $\alpha-$ stimulated cells [72] or by preventing IRF3 dimerization through direct binding to TBK1 and IKK- $\varepsilon$ in dsRNAstimulated or Newcastle virus-infected cells, respectively [73]. By contrast, TRAF3 positively regulates both the TLR- and RIG-I-dependent IRF-activating pathways [42] but negatively regulates the alternative NF- $\mathrm{KB}$ pathway [74].

\section{Why are scaffold proteins essential for NF-кB and IRF activation?}

Because early experimental evidence highlighted the essential role of NEMO in NF- $\mathrm{KB}$ activation [12], many laboratories hypothesized that NEMO connects upstream signaling molecules to the IKK complex. To address this hypothesis experimentally, a search for NEMO-interacting partners was conducted which led to the identification of many signaling molecules linked to NF-кB activation [75]. These results supported the notion that NEMO functions as a 'platform' recruiting upstream IKK-activating molecules [75]. The characterization of the post-translational 
modifications targeting NEMO provided further insight into its function in NF- $\mathrm{kB}$ signaling [76]. NEMO is phosphorylated and also is subject to non-degradative polyubiquitylation. This post-translational modification involves the internal lysine K63 of ubiquitin (i.e. K63-linked polyubiquitylation) and, in contrast to the degradative K48linked polyubiquitylation, does not promote recognition by the proteasome but rather triggers protein-protein interactions that mediate signal transduction. Recent findings demonstrate that the identity of both the E3 ubiquitin ligase and the polyubiquitylated lysine residues are stimulus specific, as recently summarized [75,76]. Although there is a general agreement on the signalinduced NEMO polyubiquitylation, and even if this modification might not only regulate IKK, but also mitogen-activated protein kinase activations in TLR- and IL-1 $\beta$-stimulated B cells [77], it is currently unclear if ubiquitylation triggers NEMO oligomerization and/or the recruitment of upstream signaling molecules harboring ubiquitin-binding domains [75]. Interestingly, in addition to being subject to non-degradative polyubiquitylation, NEMO also binds to K63-linked polyubiquitin chains through its NEMO ubiquitin-binding domain (NUB) $[78,79]$, indicating the existence of an interacting network between polyubiquitin chains and a variety of ubiquitinbinding domains found in multiple signaling molecules such as TAB2 and TAB3 which are involved in IKK activation $[80,81]$. Thus, the ability of NEMO to bind to polyubiquitinated proteins and to be polyubiquitinated itself is the mechanism which underlies the essential role of this scaffold protein for NF-кB activation [75].

Because of structural similarities between TANK, NAP1, SINTBAD and NEMO (Figure 4), the findings related to NEMO post-translational modifications might be relevant for the TBK1 and IKK- $\varepsilon$ scaffold proteins. Although TANK has not been shown to bind to K63-polyubiquitinated chains, it also is subject to phosphorylation and non-degradative polyubiquitination in a stimulus-dependent manner [21]. TBK1 and IKK- $\varepsilon$ phosphorylate TANK in macrophages and also are required for LPSmediated TANK polyubiquitylation, independently of their kinase activity. This observation indicates that TBK1 and IKK- $\varepsilon$ function not only as IRF3 and IRF7 kinases, but also as signaling molecules required to connect a yet to be identified E3 ligase for the non-degradative polyubiquitylation of their own scaffold protein [21]. Whether this modification of TANK is essential for LPS-mediated IRF3 activation remains unclear [21]. TBK1 and IKK- $\varepsilon$ also harbor a ubiquitin-like domain (ULD), adjacent to their N-terminal kinase domain and located upstream of their C-terminal coil-coil domains [82]. The ULD does not seem to bind to known ubiquitin-binding domains but is nevertheless crucial for TBK1 and IKK- $\varepsilon$ substrate recognition and kinase activity [82]. Therefore, these recent studies strongly indicate that optimal TBK1- and IKK- $\varepsilon-$ mediated phosphorylation requires distinct functional domains and highlight the importance of TANK nondegradative polyubiquitination for subsequent $I F N$ gene

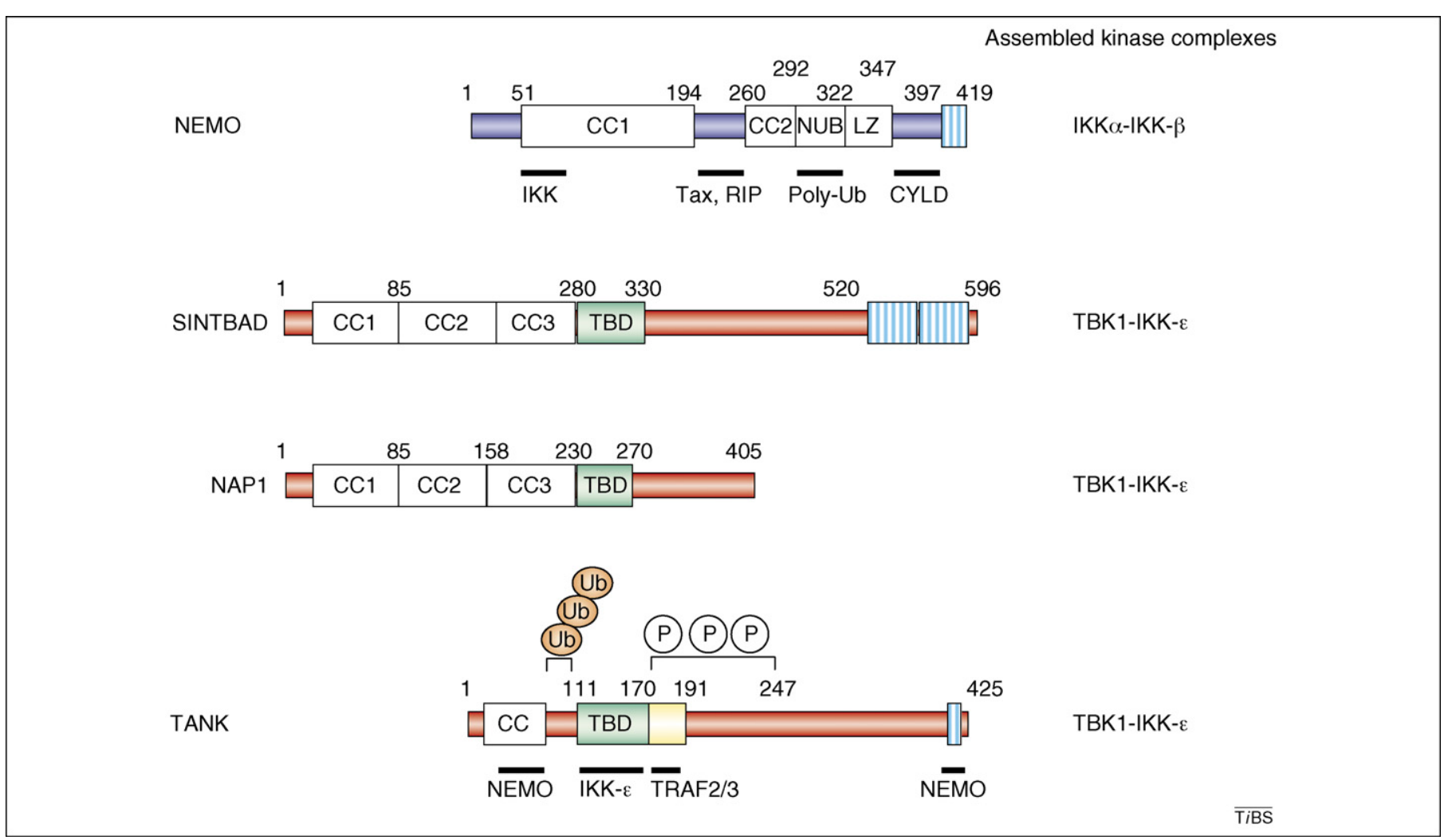

Figure 4. Structural similarities between the IKK and IKK-related scaffold proteins. The murine NEMO, SINTBAD, NAP1 and the human TANK proteins are schematically represented. The structural motifs are illustrated as boxes (CC, coil-coil; NUB, NEMO ubiquitin binding domain; LZ, leucine zipper). The domains involved in proteinprotein interactions are depicted as black bars and the interacting partners are listed below them. NEMO is represented in blue, whereas the IKK-related TBK1 and IKK- $\varepsilon$ scaffold proteins TANK, NAP1 and SINTBAD are represented in red and aligned based on their TBDs (depicted in green). The zinc finger motifs found in the C-terminal region of NEMO, SINTBAD and TANK are depicted as hatched blue rectangles. The TANK residues phosphorylated by TBK1 and IKK- $\varepsilon$ (P) are located between amino acids 171 and 247, whereas those residues targeted by the TBK1- and IKK- $\varepsilon$-dependent K63-linked polyubiquitination pathway (Ub) are located between amino acids 71 and 110. 
induction. It will be interesting to determine the extent to which the ULD found in TBK1 and IKK- $\varepsilon$ is involved in the recognition and/or the binding to TANK polyubiquitin chains.

The RIG-I CARD domain also undergoes K63-linked polyubiquitylation in response to viral infection [83]. TRIM25 is the RIG-I E3 ubiquitin ligase, and is crucial for launching the proper cellular antiviral response. Whether TRIM25 also targets other substrates, such as TANK, for polyubiquitylation, remains unknown but it is tempting to speculate that IRF3 and IRF7 activation involves sequential K63-linked polyubiquitylation of multiple scaffold proteins by potentially distinct E3 ligases.

\section{Unresolved issues \\ Do TBK1 and IKK-E function as homo- and/or heterodimers?}

Whereas it is now well established that the activation of several NF-кB-activating IKK subcomplexes is triggered by distinct stimuli exist in the cell, little information is available on the existence of distinct IKK-related complexes. Although this issue remains to be addressed experimentally, key findings might help us to speculate on the notion that TBK1 and IKK- $\varepsilon$ might, at least partially, exert their biological roles as homodimers. First, the expression levels of TBK1 and IKK- $\varepsilon$ are regulated differentially. Indeed, TBK1 is widely expressed and its level of expression is not strongly regulated at the transcription level, in contrast to IKK- $\varepsilon$, which is found in low levels unless induced by various proinflammatory cytokines [17]. Thus, these facts favor the hypothesis that TBK1 homodimers exist, especially in some cell types or in circumstances where IKK- $\varepsilon$ expression is not transcriptionally induced. Moreover, and in contrast to TBK1-deficient cells, which show strong defects in IFN induction, IKK- $\varepsilon$-deficient cells induce IFN normally; this is another piece of evidence indicating that TBK1 functions alone [36]. The existence of functional IKK- $\varepsilon$ homodimers is supported by the fact that this IKK-related kinase, but not TBK1, is recruited to the mitochondria following viral infection [84] and also by the more severe defects in IFN induction seen in TBK1 and IKK- $\varepsilon$ double-knockout versus TBK1 knockout cells [36].

\section{Do TANK, NAP1 or SINTBAD preferentially assemble}

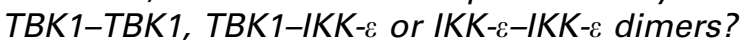

Similarly to the NF-кB-activating signaling pathways, we might expect TANK, NAP1 and SINTBAD to assemble distinct TBK1 and IKK- $\varepsilon$ complexes in a signal-specific manner. Still, it is currently unclear whether these scaffold proteins function exclusively in distinct pathways or whether they are also part of common cascades. Importantly, these proteins mainly form homo-oligomers besides some NAP1-SINTBAD hetero-oligomers. No direct association between TANK, NAP1 or SINTBAD was revealed [24]. These observations open the possibility that distinct scaffold proteins assemble some TBK1 and/or IKK- $\varepsilon$ subcomplexes. Studies addressing the subcellular localization of each scaffold protein will most likely help to resolve this issue. It is currently unknown if NAP1 and SINTBAD are targeted for non-degradative polyubiquitylation through a TBK1- and IKK- $\varepsilon$-dependent pathway but it is tempting to speculate that this mechanism is not restricted to LPS-mediated and TANK-dependent IRF3 activation. If this is indeed the case, it would be of interest to define whether a single E3 ligase targets each of them or whether some specificity occurs at that level.

\section{Are TANK, NAP1 and SINTBAD required for TBK1 and $I K K-\varepsilon$ oncogenic potential?}

Recent studies extended the roles of TBK1 and IKK- $\varepsilon$ to signaling in cancer and defined both proteins as oncogenic kinases $[85,86]$. For IKK- $\varepsilon$, this oncogenic potential is the result of an increased expression found in breast cancer samples having the amplified 1q32 locus that encompasses the $i k k-\varepsilon$ gene [85]. Interestingly, enhanced IKK- $\varepsilon$ expression in those samples is correlated with c-Rel nuclear localization [85]. It is currently unclear whether or not overexpressed IKK- $\varepsilon$ targets other substrates aside from IRF3 and IRF7, and the NF-кB proteins, and whether TANK, NAP1 or SINTBAD are required in these pathways. Because it is likely that K63-linked polyubiquitylation is required for proper $\mathrm{NF}-\kappa \mathrm{B}$ and IRF signaling, deregulation of this modification might contribute to cancer development and/or progression.

\section{Concluding remarks}

Significant progress has recently been made regarding the molecular mechanisms underlying NF-кB and IRF activation. Whereas the central roles of the IKK, TBK1 and IKK- $\varepsilon$ kinase complexes are now well established, the mechanism of their assembly is only now becoming clear. Surprisingly, similarities between the two pathways have emerged, such as the importance of signal-induced nondegradative polyubiquitylation of the constituent scaffold proteins. A thorough understanding of the physiological relevance of these post-translational modifications awaits the phenotypic characterization of mouse models in which the residues targeted for polyubiquitylation are specifically mutated ('knock-in' mice). Whereas such residues have been identified for NEMO, the targeted residues in TANK remain unknown. Future studies should tell us more on the roles of these post-translational modifications for proper signaling in innate immunity. It will also be interesting to know whether such modifications are impaired in cancer, especially in cases where TBK1 and IKK- $\varepsilon$ functions are deregulated. Importantly, numerous solid and hematological tumors have constitutive IKK activities [10]; some of these tumors also have enhanced IKK- $\varepsilon$ activity. Thus, targeting the IKKs by specific inhibitors might not be as efficient as initially thought to prevent cancer development and progression because these molecules will not target the IKK-related kinases. Thus, dissecting the TBK1 and IKK- $\varepsilon$-dependent pathways through the characterization of their interacting partners, and also through the establishment and phenotypical characterization of mouse models deficient for their scaffold proteins, will undoubtedly lead to the identification of key targets for therapeutic purposes.

\section{Acknowledgements}

We apologize to all colleagues whose papers could not be cited owing to space limitations. A.C. and J.P. are Research Associate and Research 
Director at the Belgian National Funds for Scientific Research (F.N.R.S.), respectively, whereas R.G. is a TELEVIE Research Assistant. Our laboratories are supported by grants from the F.N.R.S., TELEVIE, FWO-Vlaanderen, the Belgian Federation Against Cancer, the Concerted Research Action Program (04/09-323, University of Liege; 01G06B6, Ghent University), the Inter-University Attraction Pole 6/18 (Federal Ministry of Science), the Centre Anti-Cancéreux and the Leon Fredericq Fundation (ULg).

\section{References}

1 Akira, S. et al. (2006) Pathogen recognition and innate immunity. Cell $124,783-801$

2 Stetson, D.B. and Medzhitov, R. (2006) Type I interferons in host defense. Immunity 25, 373-381

3 West, A.P. et al. (2006) Recognition and signaling by toll-like receptors. Annu. Rev. Cell Dev. Biol. 22, 409-437

4 Doyle, S.L. and O'Neill, L. (2006) Toll-like receptors: from the discovery of $\mathrm{NF}_{\kappa} \mathrm{B}$ to new insights into transcriptional regulations in innate immunity. Biochem. Pharmacol. 72, 1102-1113

5 O'Neill, L.A. (2006) How Toll-like receptors signal: what we know and what we don't know. Curr. Opin. Immunol. 18, 3-9

6 Kato, H. et al. (2005) Cell type-specific involvement of RIG-I in antiviral response. Immunity 23, 19-28

7 Wietek, C. and O'Neill, L.A. (2007) Diversity and regulation in the NFкB system. Trends Biochem. Sci. 32, 311-319

8 Honda, K. and Taniguchi, T. (2006) IRFs: master regulators of signaling by Toll-like receptors and cytosolic pattern-recognition receptors. Nat. Rev. Immunol. 6, 644-658

9 Karin, M. and Ben-neriah, Y. (2000) Phosphorylation meets ubiquitination: the control of NF-кB activity. Annu. Rev. Immunol. 18, 621-663

10 Perkins, N.D. (2007) Integrating cell-signaling pathways with NF-кB and IKK function. Nat. Rev. Mol. Cell Biol. 8, 49-62

11 Zandi, E. et al. (1997) The IKB kinase complex (IKK) contains two kinase subunits, IKK $\alpha$ and IKK $\beta$, necessary for IкB phosphorylation and NF-кB activation. Cell 91, 243-252

12 Yamaoka, S. et al. (1998) Complementation cloning of NEMO, a component of the IkappaB kinase complex essential for NF-кB activation. Cell 93, 1231-1240

13 Rothwarf, D.M. et al. (1998) IKK- $\gamma$ is an essential regulatory subunit of the ІкB kinase complex. Nature 395, 297-300

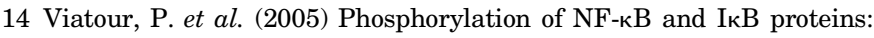
implications in cancer and inflammation. Trends Biochem. Sci. 30, 43-52

15 Tojima, Y. et al. (2000) NAK is an IкB kinase-activating kinase. Nature 404, 778-782

16 Bonnard, M. et al. (2000) Deficiency of T2K leads to apoptotic liver degeneration and impaired NF-kB-dependent gene transcription. EMBO J. 19, 4976-4985

17 Shimada, T. et al. (1999) IKK-i, a novel lipopolysaccharide-inducible kinase that is related to IKB kinases. Int. Immunol. 11, 1357-1362

18 Fitzgerald, K.A. et al. (2003) IKK $\varepsilon$ and TBK1 are essential components of the IRF3 signaling pathway. Nat. Immunol. 4, 491-496

19 Sharma, S. et al. (2003) Triggering the interferon antiviral response through an IKK-related pathway. Science 300, 1148-1151

20 Guo, B. and Cheng, G. (2007) Modulation of the interferon antiviral response by the TBK1/IKKi adaptor protein TANK. J. Biol. Chem. 282, $11817-11826$

21 Gatot, J.S. et al. (2007) Lipopolysaccharide-mediated interferon regulatory factor activation involves TBK1/IKKe-dependent Lys63linked polyubiquitination and phosphorylation of TANK/I-TRAF. J. Biol. Chem. 282, 31131-31146

22 Sasai, M. et al. (2005) Cutting edge: NF-кB-activating kinaseassociated protein 1 participates in TLR3/Toll-IL-1 homology domain-containing adapter molecule-1-mediated IFN regulatory factor 3 activation. J. Immunol. 174, 27-30

23 Sasai, M. et al. (2006) NAK-associated protein 1 participates in both the TLR3 and the cytoplasmic pathways in type I IFN induction. $J$. Immunol. 177, 8676-8683

24 Ryzhakov, G. and Randow, F. (2007) SINTBAD, a novel component of innate antiviral immunity, shares a TBK1-binding domain with NAP1 and TANK. EMBO J. 26, 3180-3190

25 Rudolph, D. et al. (2000) Severe liver degeneration and lack of NF-кB activation in NEMO/IKK $\gamma$-deficient mice. Genes Dev. 14, 854-862
26 Dejardin, E. et al. (2002) The lymphotoxin- $\beta$ receptor induces different patterns of gene expression via two NF-kB pathways. Immunity 17 , $525-535$

27 Claudio, E. et al. (2002) BAFF-induced NEMO-independent processing of NF-к B2 in maturing B cells. Nat. Immunol. 3, 958-965

28 Coope, H.J. et al. (2002) CD40 regulates the processing of NF-кB2 p100 to p52. EMBO J. 21, 5375-5385

29 Xiao, G. et al. (2001) NF-кB-inducing kinase regulates the processing of NF-кB2 p100. Mol. Cell 7, 401-409

30 Senftleben, U. et al. (2001) Activation by IKK $\alpha$ of a second, evolutionary conserved, NF-к B signaling pathway. Science 293, 1495-1499

31 Xiao, G. et al. (2004) Induction of p100 processing by NF-кB-inducing kinase involves docking ІкB kinase $\alpha(\operatorname{IKK} \alpha)$ to $\mathrm{p} 100$ and IKK $\alpha$ mediated phosphorylation. J. Biol. Chem. 279, 30099-30105

32 Beg, A.A. et al. (1995) Embryonic lethality and liver degeneration in mice lacking the RelA component of NF-кB. Nature 376, 167-170

$33 \mathrm{Li}$, Q. et al. (1999) Severe liver degeneration in mice lacking the IкB kinase 2 gene. Science 284, 321-325

$34 \mathrm{Li}$, Z.W. et al. (1999) The IKK $\beta$ subunit of IкB kinase (IKK) is essential for nuclear factor $\kappa \mathrm{B}$ activation and prevention of apoptosis. J. Exp. Med. 189, 1839-1845

35 Tanaka, M. et al. (1999) Embryonic lethality, liver degeneration, and impaired NF-к B activation in IKK- $\beta$-deficient mice. Immunity 10, $421-429$

36 Hemmi, H. et al. (2004) The roles of two IкB kinase-related kinases in lipopolysaccharide and double stranded RNA signaling and viral infection. J. Exp. Med. 199, 1641-1650

37 McWhirter, S.M. et al. (2004) IFN-regulatory factor 3-dependent gene expression is defective in Tbk1-deficient mouse embryonic fibroblasts. Proc. Natl. Acad. Sci. U. S. A. 101, 233-238

38 Yamamoto, M. et al. (2002) A novel Toll/IL-1 receptor domaincontaining adapter that preferentially activates the IFN- $\beta$ promoter in the Toll-like receptor signaling. J. Immunol. 169, 6668-6672

39 Oshiumi, H. et al. (2003) TICAM-1, an adaptor molecule that participates in Toll-like receptor 3-mediated interferon- $\beta$ induction. Nat. Immunol. 4, 161-167

40 O'Neill, L.A. and Bowie, A.G. (2007) The family of five: TIR-domaincontaining adaptors in Toll-like receptor signaling. Nat. Rev. Immunol. $7,353-364$

41 Hoebe, K. and Beutler, B. (2006) TRAF3: a new component of the TLRsignaling apparatus. Trends Mol. Med. 12, 187-189

42 Saha, S.K. and Cheng, G. (2006) TRAF3: a new regulator of type I interferons. Cell Cycle 5, 804-807

43 Hacker, H. et al. (2006) Specificity in Toll-like receptor signaling through distinct effector functions of TRAF3 and TRAF6. Nature 439, 204-207

44 Oganesyan, G. et al. (2006) Critical role of TRAF3 in the Toll-like receptor-dependent and -independent antiviral response. Nature 439, 208-211

45 Kawai, T. and Akira, S. (2006) Innate immune recognition of viral infection. Nat. Immunol. 7, 131-137

46 Kawai, T. et al. (2004) Interferon- $\alpha$ induction through Toll-like receptors involves a direct interaction of IRF7 with Myd88 and TRAF6. Nat. Immunol. 5, 1061-1068

47 Hoshino, K. et al. (2006) IкB kinase- $\alpha$ is critical for interferon- $\alpha$ production induced by Toll-like receptors 7 and 9. Nature 440, 949-953

48 Seth, R.B. et al. (2005) Identification of MAVS, a mitochondrial antiviral signaling protein that activates NF- $\mathrm{BB}$ and IRF 3. Cell $122,669-682$

49 Kawai, T. et al. (2005) IPS-1, an adaptor trigerring RIG-I- and Mda5mediated type I interferon induction. Nat. Immunol. 6, 981-988

$50 \mathrm{Xu}$, L.G. et al. (2005) VISA is an adapter protein required for virustriggered IFN- $\beta$ signaling. Mol. Cell 19, 727-740

51 Meylan, E. et al. (2005) Cardif is an adaptor protein in the RIG-I antiviral pathway and is targeted by hepatitis C virus. Nature 437 , $1167-1172$

52 Ishii, K.J. et al. (2006) A Toll-like receptor-independent antiviral response induced by double-stranded B-form DNA. Nat. Immunol. 7, $40-48$

53 Takaoka, A. et al. (2007) DAI (DLM-1/ZBP1) is a cytosolic DNA sensor and an activator of innate immune response. Nature 448, 501-506

54 Cheng, G. and Baltimore, D. (1996) TANK, a co-inducer with TRAF2 of TNF- and CD 40L-mediated NF-кB activation. Genes Dev. 10, 963-973 
55 Kaye, K.M. et al. (1996) Tumor necrosis factor receptor associated factor 2 is a mediator of NF-kB activation by latent infection membrane protein 1, the Epstein-Barr virus transforming protein. Proc. Natl. Acad. Sci. U. S. A. 93, 11085-11090

56 Rothe, M. et al. (1996) I-TRAF is a novel TRAF-interacting protein that regulates TRAF-mediated signal transduction. Proc. Natl. Acad. Sci. U. S. A. 93, 8241-8246

57 Chariot, A. et al. (2002) Association of the adaptor TANK with the I кB kinase (IKK) regulator NEMO connects IKK complexes with IKK $\varepsilon$ and TBK1 kinases. J. Biol. Chem. 277, 37029-37036

58 Bonif, M. et al. (2006) TNF $\alpha$ - and IKK $\beta$-mediated TANK/I-TRAF phosphorylation: implications for interaction with NEMO/IKK $\gamma$ and NF-кB activation. Biochem. J. 394, 593-603

59 Adli, M. and Baldwin, A.S. (2006) IKK-i/IKK $\varepsilon$ controls constitutive, cancer cell-associated NF- $\mathrm{B}$ activity via regulation of Ser-536 p65/ RelA phosphorylation. J. Biol. Chem. 281, 26976-26984

60 Mattioli, I. et al. (2006) Inducible phosphorylation of NF-к B p65 at serine 468 by $\mathrm{T}$ cell costimulation is mediated by IKKع. J. Biol. Chem. 281, 6175-6183

61 Harris, J. et al. (2006) Nuclear accumulation of cRel following Cterminal phosphorylation by TBK1/IKKE. J. Immunol. 177, 2527-2535

62 Wietek, C. et al. (2006) IкB kinase $\varepsilon$ interacts with p52 and promotes transactivation via p65. J. Biol. Chem. 281, 34973-34981

63 Nomura, F. et al. (2000) NF-кB activation through IKK-i-dependent ITRAF/TANK phosphorylation. Genes Cells 5, 191-202

64 Pomerantz, J.L. and Baltimore, D. (1999) NF-кB activation by a signaling complex containing TRAF2, TANK and TBK1, a novel IKK-related kinase. EMBO J. 18, 6694-6704

65 Fujita, F. et al. (2003) Identification of NAP1, a regulatory subunit of IкB kinase-related kinases that potentiates NF-кB signaling. Mol. Cell. Biol. 23, 7780-7793

66 Schreiber, J. et al. (2006) Coordinated binding of NF-кB family members in the response of human cells to lipopolysaccharide. Proc. Natl. Acad. Sci. U. S. A. 103, 5899-5904

67 Zhao, T. et al. (2007) The NEMO adaptor bridges the nuclear factor-кB and interferon regulatory factor signaling pathways. Nat. Immunol. 8, $592-600$

68 Wietek, C. et al. (2003) Interferon regulatory factor-3-mediated activation of the interferon-sensitive response element by Toll-like receptor (TLR) 4 but not TLR3 requires the p65 subunit of NF-к. J. Biol. Chem. 278, 50923-50931

69 Ogawa, S. et al. (2005) Molecular determinants of crosstalk between nuclear receptors and toll-like receptors. Cell 122, 707-721
70 Leung, T.H. et al. (2004) One nucleotide in a $\mathrm{\kappa B}$ site can determine cofactor specificity for NF-kB dimers. Cell 118, 453-464

71 Wietek, C. et al. (2006) IкB kinase $\varepsilon$ interacts with p52 and promotes transactivation by p65. J. Biol. Chem. 281, 34973-34981

72 Wertz, I.E. et al. (2004) De-ubiquitination and ubiquitin ligase domains of A20 downregulate NF-кB signaling. Nature 430, 694-699

73 Saitoh, T. et al. (2005) A20 is a negative regulator of IFN regulatory factor 3 signaling. J. Immunol. 174, 1507-1512

74 Hauer, J. et al. (2005) TNF receptor (TNFR)-associated factor (TRAF) 3 serves as an inhibitor of TRAF2/5-mediated activation of the noncanonical NF-кB pathway by TRAF-binding TNFRs. Proc. Natl. Acad. Sci. U. S. A. 102, 2874-2879

75 Sebban, H. et al. (2006) Posttranslational modifications of NEMO and its partners in NF-кB signaling. Trends Cell Biol. 16, 569-577

76 Israel, A. (2006) NF-кB activation: nondegradative ubiquitination implicates NEMO. Trends Immunol. 27, 395-397

77 Yamamoto, M. et al. (2006) Key function for the Ubc13 E2 ubiquitinconjugating enzyme in immune receptor signaling. Nat. Immunol. 7, 962-970

$78 \mathrm{Wu}$, C.J. et al. (2006) Sensing of Lys 63-linked polyubiquitination by NEMO is a key event in NF- $\mathrm{BB}$ activation. Nat. Cell Biol. 8, 398406

$79 \mathrm{Ea}, \mathrm{C} . \mathrm{K}$. et al. (2006) Activation of IKK by TNF $\alpha$ requires site-specific ubiquitination of RIP1 and polyubiquitin binding by NEMO. Mol. Cell $22,245-257$

80 Ishitani, T. et al. (2003) Role of the TAB2-related protein TAB3 in IL-1 and TNF signaling. EMBO J. 22, 6277-6288

81 Kanayama, A. et al. (2004) TAB2 and TAB3 activate the NF-кB pathway through binding to polyubiquitin chains. Mol. Cell 15, 535548

82 Ikeda, F. et al. (2007) Involvement of the ubiquitin-like domain of TBK1/IKK-i kinases in regulation of IFN-inducible genes. EMBO J. 26 , 3451-3462

83 Gack, M.U. et al. (2007) TRIM25 RING-finger E3 ubiquitin ligase is essential for RIG-I-mediated antiviral activity. Nature 446, 916-920

84 Lin, R. et al. (2006) Dissociation of a MAVS/IPS-1/VISA/Cardif-IKK $\varepsilon$ molecular complex from the mitochondria outer membrane by hepatitis C virus NS3-4A proteolytic cleavage. J. Virol. 80, 6072-6083

85 Boehm, J.S. et al. (2007) Integrative genomic approaches identify IKBKE as a breast cancer oncogene. Cell 129, 1065-1079

86 Chien, Y. et al. (2006) RalB GTPase-mediated activation of the IкB family kinase TBK1 couples innate immune signaling to tumor cell survival. Cell 127, 157-170

\section{Reproduction of material from Elsevier articles}

Interested in reproducing part or all of an article published by Elsevier, or one of our article figures? If so, please contact our Global Rights Department with details of how and where the requested material will be used. To submit a permission request online, please visit:

www.elsevier.com/locate/permissions 